\author{
Military Technical College \\ Kobry El-Kobbah, \\ Cairo, Egypt.
}

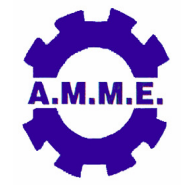

\title{
FAULTS DIAGNOSIS USING ON-SHAFT VIBRATION MEASUREMENT IN ROTATING MACHINES
}

$15^{\text {th }}$ International Conference
on Applied Mechanics and
Mechanical Engineering.

\author{
M. E. Elnady*, J. K. Sinha** and S. O. Oyadiji**
}

\begin{abstract}
Rotating machines are an essential element for most of the modern mechanical systems. Hence, the reliable and continuous monitoring of their health is always receiving attention to ensure safety and reduce downtime. Vibration based fault diagnosis is one of the robust and well established techniques used to monitor the condition of a rotating machine. The early the fault is detected the more time is allowed for proper diagnosis and maintenance planning. The conventional method of measuring the vibration on the bearing pedestals proved to be applicable in most cases of machines structures and configurations. However, it requires a number of vibration sensors at each bearing pedestal for fault diagnosis. The on-shaft vibration measurement forms a proper solution as it provides enriched vibration content with much less number of sensors, and consequently leads to better fault diagnosis. In this paper, the on-shaft vibration measurement technique is presented and its applicability to diagnose different faults introduced to an experimental test rig is investigated.
\end{abstract}

\section{KEY WORDS}

Condition monitoring, rotating machines, on-shaft vibration measurement, faults diagnosis.

Graduate student, school of MACE, the University of Manchester, Manchester M13 9PL, U.K. Email: maged.elnady@gmail.com.

** School of MACE, the University of Manchester, Manchester M13 9PL, U.K. Email: jyoti.Sinha@manchester.ac.uk.

*** Lecturer, school of MACE, the University of Manchester, Manchester M13 9PL, U.K. Email:s.o.oyadiji@manchester.ac.uk. 


\section{INTRODUCTION}

\section{Condition Monitoring of Rotating Machines}

Rotating machines form a major component of almost all industrial facilities and their maintenance is of great importance. The vibration-based condition monitoring of rotating machines starts with acquiring as much as possible vibration data from the machine and continuously monitoring the changes in some selected parameters depending on the used analysis technique (time, frequency, time-frequency,...). After that, appropriate analysis is done to monitor their behaviour along time. Continuous monitoring can lead to early diagnosis of incipient faults [1]. These achievements lead at the end to reduced maintenance costs and minimized machine failure hazards. Among the many references available, De Silva [2] dedicated a chapter for machine condition monitoring and fault diagnostics and Mobley [3] discussed condition monitoring in a predictive maintenance framework.

The vibration sensor location is selected to give the maximum signal-to-noise $(\mathrm{s} / \mathrm{n})$ ratio. Being the nearest stationary part to the vibration source (shaft, bearing, gears...), the bearing pedestals are considered as the most suitable position for an accelerometer. However, depending on the machine configuration, bearing pedestals might not be the best sensor position and direct measurement of the shaft vibration is recommended.

To measure the shaft vibration directly, a proximity probe attached to bearing pedestal can be used in conjunction with an accelerometer on the pedestal. To avoid the proximity sensor limitations and get closer to the shaft, a strain gage may be attached to the shaft with a telemetry unit to transmit the measurements to a receiver unit [4]. Many available commercial kits are based on this concept and they even enable multi sensor measurements. They can measure many parameters such as temperature, pressure, torque, etc.

The recent developments in Micro Electro Mechanical Systems (MEMS) and miniaturized wireless communication devices gave the opportunity to mount a MEMS accelerometer, a power source, and data acquisition and transmission equipments on a rotating structure without altering its dynamic characteristics [5]. Their small weight hardly affects the rotor balance and the low power consumption enables extended operating times before the need for battery recharge.

\section{On-Shaft Vibration Measurement}

Taking the measurement on the rotor means that the measurements are done in a coordinates system that is already rotating with the same speed of the rotor. Bejarano et al. [6] made use of this important feature and treated a rotating cracked shaft problem as the well known stationary cracked beam one. They successfully detected rotor crack.

In a vibration control context, Sloetjes and De Boer [7] used a strain gage attached to an accelerating rotor to measure the overall vibration. They used it to control a 
piezocermaic batch bonded to the rotor to enhance the rotor stability. They used a slip ring assembly to communicate with the devices on the rotor.

Rather than using the overall vibration, its spectral components are known to be more informative and can help identifying the fault source. Arebi et al. [8] used an on-shaft accelerometer and an encoder to measure the tangential acceleration and the Instantaneous Angular Speed (IAS) of a misaligned rotor respectively. They monitored the changes in harmonics amplitudes with speed and misalignment severity. The signal obtained from the on-shaft accelerometer detected misalignment more efficiently than those from the ordinary encoder. However, they noticed incomplete conformance between the trends of the harmonics from the accelerometer and those from the encoder.

Considering the measurement system components, Arms et al. [9] assembled a strain measurement system and installed it on a helicopter pitch link for health monitoring. The system comprises energy harvesting and storage units to power a microcontroller and a wireless transmitter.

\section{Pattern Classification}

Fault detection may be defined as the departure of a measured parameter from a range that is known to represent normal operation [2]. One or more parameters can be used to accurately describe the machine state. A variety of parameters from different domains (time, frequency, ...) were investigated $[2,10,11]$.

For the basic detection level total machine vibration is used for comparison; the accumulated experience produced standards (ISO 10816-3) from which safe ranges are extracted. However, for the more advanced level of using processed parameters (rather than the total machine vibration); limits are customized for each machine and analysis types. For example, when using a vibration spectrum to monitor different machine harmonics, a threshold is set for each one of the major spectral lines. An alarm is triggered when one of the spectral lines exceeds its preset threshold. This advanced method gives some guidance to the subsequent step of fault diagnosis since it relates the spectral line that triggered the alarm to its generating component in the machine.

Early detection of faults gives more time to plan machine shutdown. More importantly, it gives more time for fault diagnosis. Fault diagnosis may be defined as a procedure of mapping the information obtained in the measurement space and/or features in the feature space to machine faults in the fault space [2].

An experienced analyst can do the mapping manually. Many researchers reported how do different faults manifest themselves in different domains [10-12]. To avoid the need for experienced analysts and benefit from the advantages of fast computing and large databases management, a computer can be used to do the process of automatic mapping or in other words "pattern classification". In the pattern classification process, features representing the machine state are gathered to form a feature vector, which is then compared against a set of feature vectors representing different machine states (a training set). In this work, the investigated machine state is classified using the nearest neighbour classifier. 
The on-shaft vibration measurement is not yet generalized to diagnosis different faults in rotating machines. To benefit from the standardized faults diagnosis techniques; we need to make sure that it responds to different machine conditions/faults in the same way as the on-bearing one does. Up till now, the onshaft vibration harmonics were not thoroughly investigated nor compared to the onbearing ones. In this work, the on-shaft vibration measurement method is thoroughly investigated in time, frequency and order domains. Following that, the capability to diagnose different common rotating machines faults is benchmarked using that of the conventional on-bearing method. An experimental test rig is used to investigate an on-shaft vibration measurement system and to simulate faults.

The rest of this paper constitutes four sections. In section two, the experimental test rig is described and modal testing results are shown. In section three, an exploratory run up is performed and analyzed in different domains where a mismatching between the on-bearing and on-shaft signals is reported. This called for the pattern classification approach to diagnose faults, which is discussed in section four.

\section{TEST RIG}

\section{Description}

The test rig consists of a $20 \mathrm{~mm}$ diameter shaft with a span of $900 \mathrm{~mm}$ supported on a relatively rigid foundation through ball bearings, see Figure 1 . The shaft also carries a balanced disc of $125 \mathrm{~mm}$ diameter and $20 \mathrm{~mm}$ thickness at mid span. A motor is connected to the shaft through a flexible coupling.

Figure 2 shows the proposed vibration measurement scheme. A small tiny micro electro-mechanical system (MEMS) accelerometer is mounted on the shaft itself (not shown in figure). The accelerometer has a range of ${ }_{ \pm} 40 \mathrm{~g}$ and sensitivity of about $200 \mathrm{mV} / \mathrm{g}$. It is assumed that the mounting of such a tiny accelerometer may not influence the rotor unbalance. The V-Link ${ }^{\circledR}$ from MicroStrain ${ }^{\circledR}$ is the wireless sensor node which is then connected to the MEMS accelerometer for wireless transmission of the vibration signals. The V-Link module weighs just $97 \mathrm{gm}$ and uses a 12 bit analogue to digital converter with data sampling rates of $736 \mathrm{~Hz}$ and $2048 \mathrm{~Hz}$ in streaming and data logging modes respectively.

\section{Modal Testing}

Modal testing was done on the rig by the Impulse-Response method [13] to identify its natural frequencies. A typical Frequency Response Function (FRF) plot is shown in Figure 3. The peaks at 33 and $35.5 \mathrm{~Hz}$ are identified as the first two critical speeds. With these observations the run-up experiment has been conducted which is discussed in the next section.

\section{RUN UP}

A run-up experiment up to a shaft speed of 1200 RPM with a linear rate of 4.3 $\mathrm{RPM} / \mathrm{s}$ has been conducted. The acceleration vibration data has also been collected 
at bearing 1 as well as from the on-shaft accelerometer. The following subsections describe vibration signal analysis in different domains.

\section{Time Waveform Analysis}

Figure 4 shows the time history for all signals. It is clear that response amplification is observed as the rig passes through integer fractions of its critical speeds. Comparing the on-bearing and on-shaft signals, we can conclude that the on-shaft measurement had captured almost all the peaks noticed in the on-bearing signals with a much higher $\mathrm{s} / \mathrm{n}$ ratio. On the other side it's highly affected by gravity. The gravity effect appears as an oscillation with amplitude of $1 \mathrm{~g}$ and a synchronous frequency.

\section{Spectral Analysis}

The time waveforms did not reveal the spectral content, therefore the Short Time Fourier Transformation (STFT) analysis was carried out. Typical spectrogram for the on-bearing signal is shown in Figure 5. It shows frequency peaks related to the shaft speed $(1 \mathrm{x})$ and its higher harmonics $(2 \mathrm{x}, 3 \mathrm{x} \ldots)$ as expected.

Additionally, the transient amplitude amplifications noticed in the time waveforms appear as distinct spots observed when the shaft speed passes through 1/3, 1/4 and $1 / 2$ of the critical speeds. Another observation in the spectrogram is that increased background noise is found below the speed of $450 \mathrm{RPM}$. This is referred to a change in the carrier frequency of the $A C$ voltage driving the motor. This change was clearly audible during the experiments.

With these observations, the STFT analysis has also been carried out for the onshaft signal, see Figure 5. As expected, the spectrogram shows the strong feature of $1 \mathrm{x}$ component due to gravity. There is no sign for the noise coming from the motor below 450 RPM, which depicts the on-shaft measurement immunity to noise from surrounding machines.

Examining the transient response peaks, instead of showing a single peak when the rotor passes an integer fraction of a certain critical speed, two peaks are there. This is interpreted as an amplitude modulation affecting the on-shaft signal due to the rotational motion of the sensor.

\section{Order Analysis}

In overall, the time waveform and spectral analyses and the comparison between onshaft and on-bearing vibration measurements suggest the following features for the on-shaft measurement scheme:

- An interestingly higher $\mathrm{s} / \mathrm{n}$ ratio

- Immunity to structural noise from surrounding sources.

In spite of these encouraging features, the following problems are noticed:

- Signal modulation with the rotational speed.

- Dominant gravity effect.

This draws doubts to the mechanism generating the on-shaft harmonics and consequently their applicability in faults diagnosis and rotor dynamics analyses in 
general. In this section, additional investigation is done to examine the conformance between on-shaft and on-bearing harmonics. The behaviour of the on-shaft harmonics $(1 \mathrm{x}, 2 \mathrm{x}, 3 \mathrm{x}$...) upon changing speed is investigated.

The run up discussed earlier is reviewed from another perspective. We previously computed the STFT for a time domain signal acquired at constant time intervals (i.e. $\Delta t=$ constant). This generated a colour map describing the relation between frequency and time (or speed, since they are linearly related according to the ramp). That plot makes it difficult to extract rotational speed harmonics since they are represented by straight lines starting at origin and having different slopes $(1,1 / 2$, $1 / 3 \ldots$..). To make the harmonics extraction much easier, we can transform the run up data from the time domain to the angular domain [14], where the data is sampled at constant rotational angles (i.e. $\Delta \theta=$ constant). Upon resampling the time waveform at constant angles, the STFT is computed and different harmonics could be easily extracted. The first four harmonics are extracted and plotted against speed, see Figure 6. As expected, the on-bearing $2 x, 3 x$ and $4 x$ harmonics show peaks at $1 / 2$, $1 / 3$ and $1 / 4$ the critical speeds respectively.

The situation is different for the on-shaft harmonics; some of them (e.g. $1 \mathrm{x}$ and $3 \mathrm{x}$ ) share some peaks. Also, some of them comprise peaks belonging to more than one of the on-bearing harmonics. For example, the on-shaft $3 x$ includes peaks from both on-bearing $2 x$ (at $1 / 2$ the critical speeds) and $4 x$ (at $1 / 4$ the critical speeds). We can also notice the gravity effect in the on-shaft $1 x$ harmonic; the whole plot is elevated by $1 \mathrm{~g}$.

This strong correlation among on-shaft harmonics, in addition to the inherent modulation, necessitates automatic fault classification instead of the manual one. This is discussed in the following section.

\section{FAULTS DIAGNOSIS}

\section{Description of Machine States/Faults}

In this work, common machine faults; misalignment, unbalance and looseness (in addition to a reference state) are considered, see Table 1. To introduce misalignment, the outboard bearing pedestal is translated (from its position in the reference state) by increments of $0.6 \mathrm{~mm}$ in the horizontal plane. Unbalance is done by adding small masses to the disc; 3 grams for each one of the two unbalance levels. For more accurate evaluation, looseness is introduced by loosening the unbalance weight itself (at the second unbalance level). This is expected to let the looseness case be very similar to the unbalance one. The combined fault case is achieved by introducing unbalance and misalignment simultaneously.

A set of experiments is conducted where faults are seeded to the rig and a classification algorithm is applied to diagnose the faults. Since the main purpose is to compare the fault diagnosis capabilities on a fair basis, a simple classification procedure is followed. 


\section{Nearest Neighbour Classifier}

Figure 7 summarizes the developed classification algorithm. After segmenting the acquired vibration samples into a number of observations, they are divided into training and testing sets. For each observation, a feature vector is constructed from different signal attributes. The attributes can be computed in different domains to fully and accurately describe the signal, see Table 2. Feature normalization is applied to handle the variation of units in the signal features.

The idea of the nearest neighbour classifier is to search all the training observations to find the nearest one (or $k$ nearest ones for the generalized case) to the investigated observations. The comparison measure is the Euclidean distance between each two examined feature vectors.

In pattern classification, it is known that the higher the number of features used is, the higher the size of training set should be. This is why a dimension reduction step is needed. To reduce the number of features (initially twelve), many techniques are available, such as Principal component Analysis [15]. However, in this work, a very simple method is used to assess the features quality through a dimensionless Quality Index (QI).

Clearly a good feature should posses the following two criteria:

- Minimum variance among observations taken at the same machine state.

- Maximum change upon changes in the machine state.

So if we group observations into clusters based on their respective machine states, and calculate the clusters means and variances, we can achieve the above mentioned quality criteria by expressing (QI) as:

$$
Q I=\frac{V O M}{M O V}
$$

Where $V O M$ is the variance of the mean values of all clusters and MOV is the mean value of the clusters variances.

After calculating (QI) for all features, the three with highest (QI) can be extracted and fed into the classifier.

\section{Results}

The rig is run at a constant speed (1200 RPM) and vibration data is sampled at 500 $\mathrm{Hz}$. The acquisition lasted for five minutes and data were split into thirty two observations per machine state. For cross validation, the observations are randomly divided into training (twenty observations) and testing (twelve observations) sets. The testing set is held out to never affect the training process. The classification process is repeated for twenty times and the average results are recorded.

The features vectors are calculated for all observations and the proposed feature quality index method is used to select the best features and reject the bad ones. For example, Figure 8 shows the quality indices for features describing the vibration signals and Figure 9 depicts the efficiency of this feature selection method by 
contrasting high and low quality features. The higher quality feature shows higher stability within the same state and more separation among different states.

The classification is performed in two phases, an initial one to examine the capability of fault detection and a subsequent one to examine the capability of fault severity assessment. For preliminary fault detection, the machine state is considered to be absolutely unknown. Hence, the observations under test are compared against all known machine states.

For misalignment and unbalance faults, a generalized state is used for fault detection. That is done by combining all the misalignment cases (M1-M3) and the unbalance cases (U1-U2) into two general cases; (M) and (U) respectively, as shown in Table 1.

Figure 10 shows the classification results for the fault detection phase. The on-shaft measurement shows higher reliability since more observations within each testing set are correctly classified. The results show that the on-shaft measurement successfully classified all observations except for set number six, while the onbearing one got confused distinguishing the unbalance and looseness faults. This is because of the great physical similarity between them (as described earlier). In the next phase, all of the misalignment, unbalance, and looseness sets are forwarded to the fault level assessment round. For that purpose, they are going to be compared against the training sets for each one of their respective faults separately.

Figure 11 and Figure 12 present the fault level assessment results. They show that the on-shaft method outperformed the on-bearing one in assessing the unbalance fault level and in distinguishing the two close faults of unbalance and looseness. The accuracies of both methods in the misalignment fault level assessment are nearly equal and acceptable.

\section{CONCLUSIONS}

On-shaft vibration measurement has been investigated and applied to a laboratory scale rotating machine model. The measured vibration has been compared to the on-bearing one in different domains. It appeared to have modulation with the rotational speed and dominant gravity effect. However it contains enriched vibration information compared to that acquired on the bearing pedestals.

The proposed approach has been also applied to diagnose different faults commonly found in rotating machines. The PCA is used to reduce the dimensions of the fault features vector and the nearest neighbour classifier is used to classify different machine states. The fault diagnosis results suggest that the on-shaft vibration gives better diagnosis efficiency compared to the on-bearing one. Future work is planned for more detailed investigation of the on-shaft vibration measurement and also for the diagnosis of more faults.

\section{ACKNOWLEDGMENT}

M.E. Elnady is grateful to the Egyptian Ministry of Defence for funding this project 
through grant 2623/02. The authors would like to thank the workshop technical staff at the school of MACE, University of Manchester for their effort manufacturing the rig parts.

\section{REFERENCES}

[1] Jardine, A. K. S., Lin, D., and Banjevic, D., 2006, "A review on machinery diagnostics and prognostics implementing condition-based maintenance," Mechanical Systems and Signal Processing, 20(7), pp. 1483-1510.

[2] De Silva, C. W., Vibration and shock handbook, CRC Press (27 Jun 2005).

[3] Mobley, K. R., 2002, An introduction to predictive maintenance, ButterworthHeinemann.

[4] Filho, A. C. L., Belo, F. A., Santos, J. L. A. d., and Anjos, E. G. d., 2011, "SelfPowered Telemetric Torque Meter," Journal of Dynamic Systems, Measurement, and Control, 133(4), p. 045001.

[5] Elnady, M. E., Sinha, J. K., and Oyadiji, S. O., 2011, "On-Shaft Wireless Vibration Measurement for Condition Monitoring of Rotating Machines," 10th biennial International Conference on Vibration Problems, PRAGUE, Czech Republic.

[6] Bejarano, F. A., Jia, Y., and Just, F., 2009, "Crack Identification of A Rotating Shaft with Integrated Wireless Sensor," International Journal On Smart Sensing and Intelligent Systems, 2(4), pp. $564-578$.

[7] Sloetjes, P., and de Boer, A., 2008, "Vibration reduction and power generation with piezoceramic sheets mounted to a flexible shaft," Journal of Intelligent Material Systems and Structures, 19(1), pp. 25-34.

[8] Arebi, L., Gu, F., Hu, N., and Ball, A., 2011, "Misalignment Detection Using a Wireless Sensor Mounted on a Rotating Shaft," International congress on condition monitoring and diagnostic engineering management, pp. 12891299.

[9] Arms, S. W., Townsend, C. P., Churchill, D. L., Moon, S. M., and Phan, N., 2006, "Energy harvesting wireless sensors for helicopter damage tracking," Proc. AHS International Forum 62, HUMS III session.

[10] Randall , R. B., 2004, "State of the art in monitoring rotating machine-part1," Sound and vibration.

[11] Randall, R. B., 2004, "State of the art in monitoring rotating machine-part2," Sound and vibration.

[12] Edwards, S., Lees, A. W., and Friswell, M. I., 1998, "Fault diagnosis of rotating machines," The shock and vibration digest, 30(1), pp. 4-13.

[13] Ewins, D. J., 2000, Modal Testing - Theory, Practice and Application, Research Studies Press, U.K.

[14] Groover, C., Trethewey, M., Maynard, K., and Lebold, M., 2005, "Removal of order domain content in rotating equipment signals by double resampling," Mechanical Systems and Signal Processing, 19(3), pp. 483-500.

[15] Zimroz, R., and Bartkowiak, A., 2011, "Investigation on Spectral Structure of Gearbox Vibration Signals by Principal Component Analysis for Condition Monitoring Purposes," Journal of Physics: Conference Series, 305(1), p. 012075. 


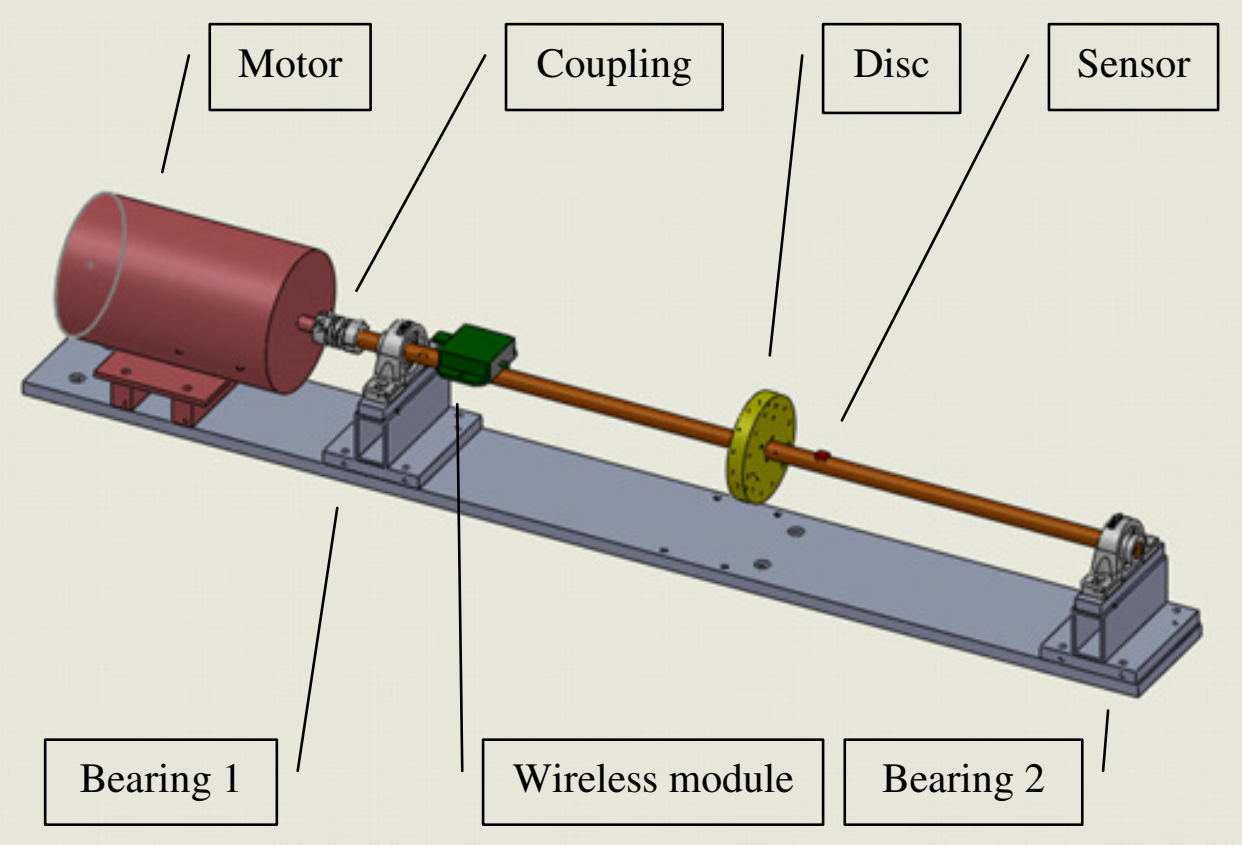

Figure 1: Test rig.

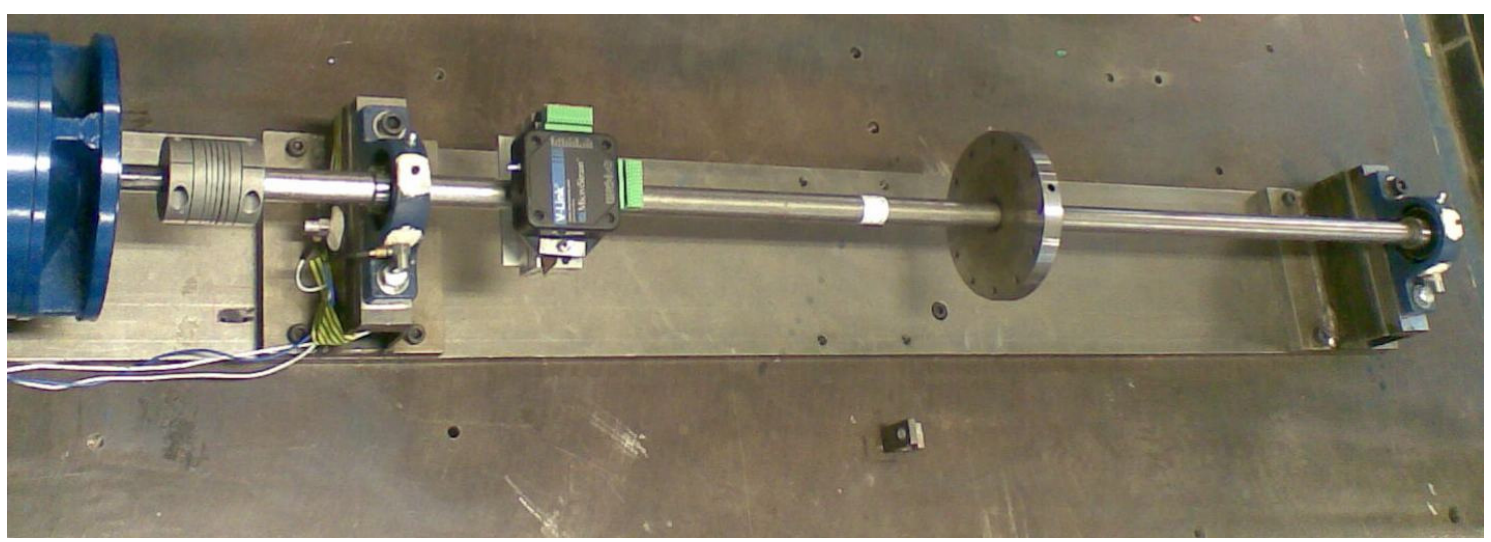

Figure 2: Rig photograph showing the vibration measurement scheme. 


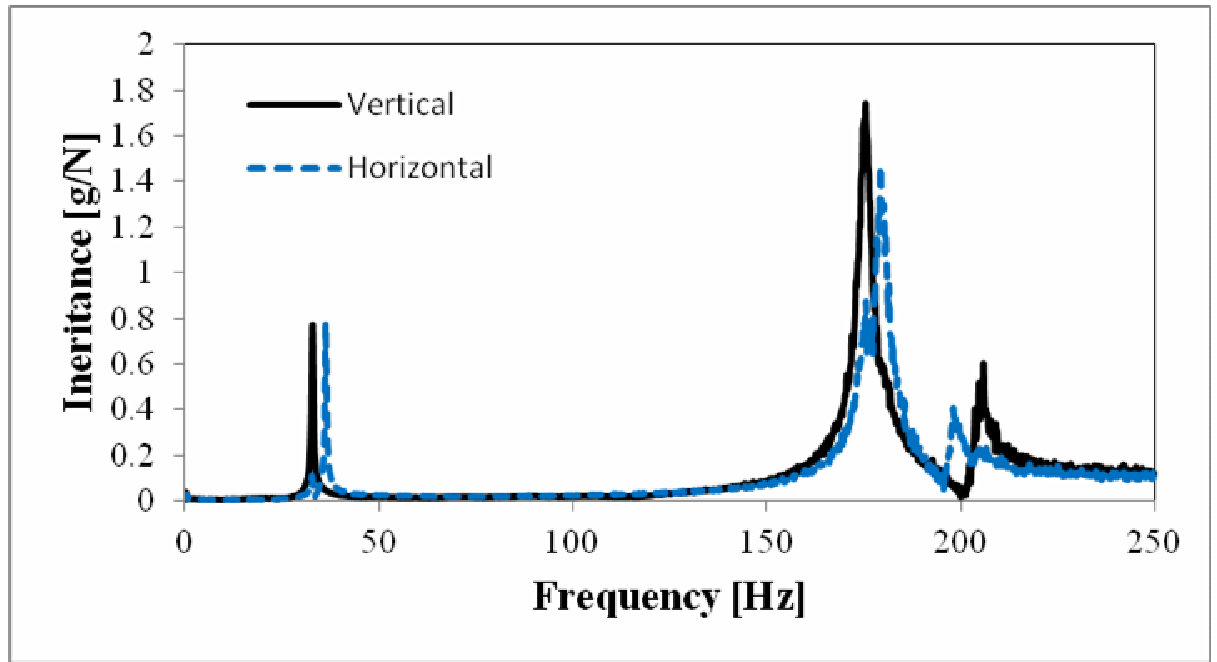

Figure 3: FRF amplitude for the experimental rig modal testing.
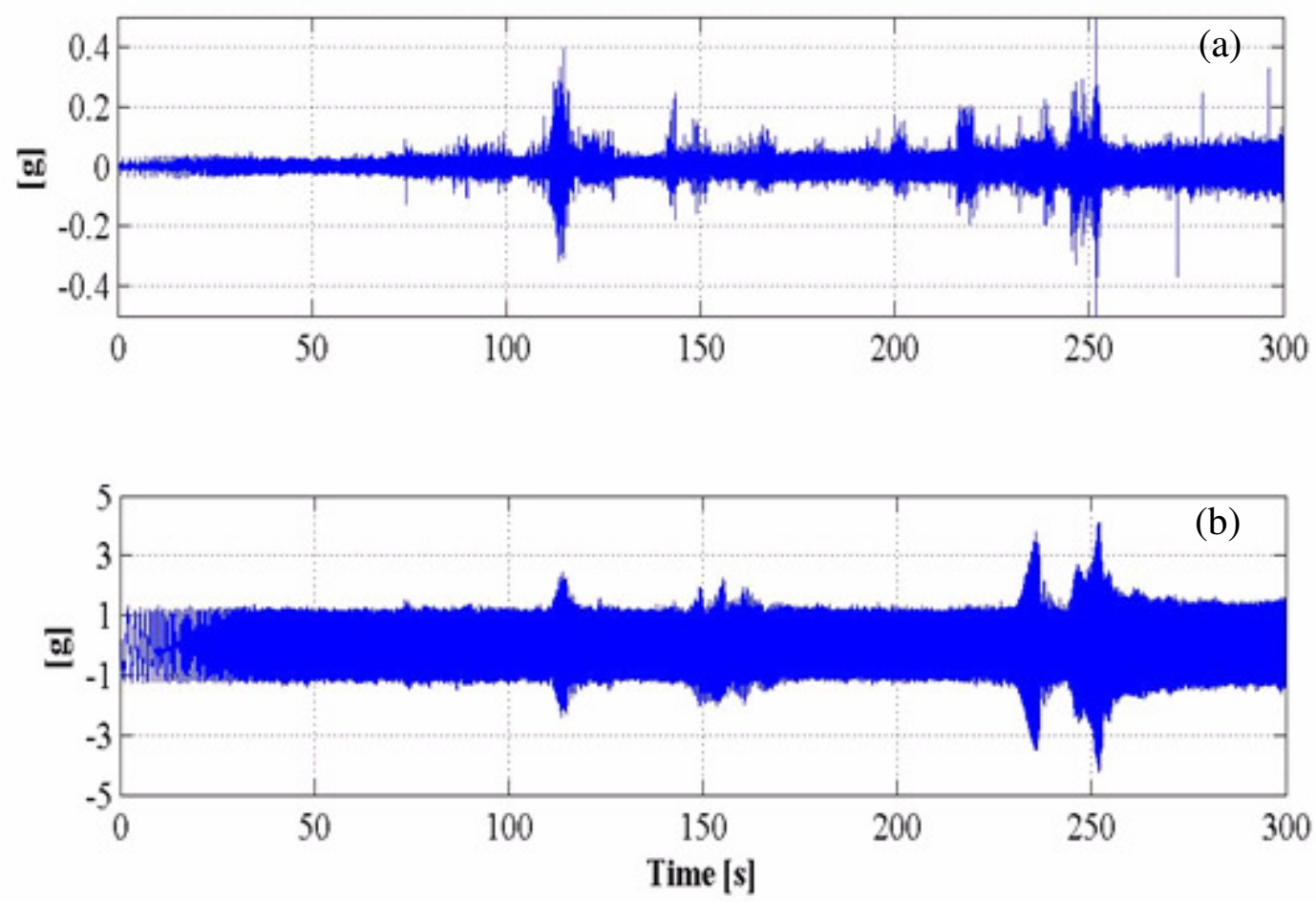

Figure 4: Measured vibration during run-up (a) on-bearing and (b).on-shaft 

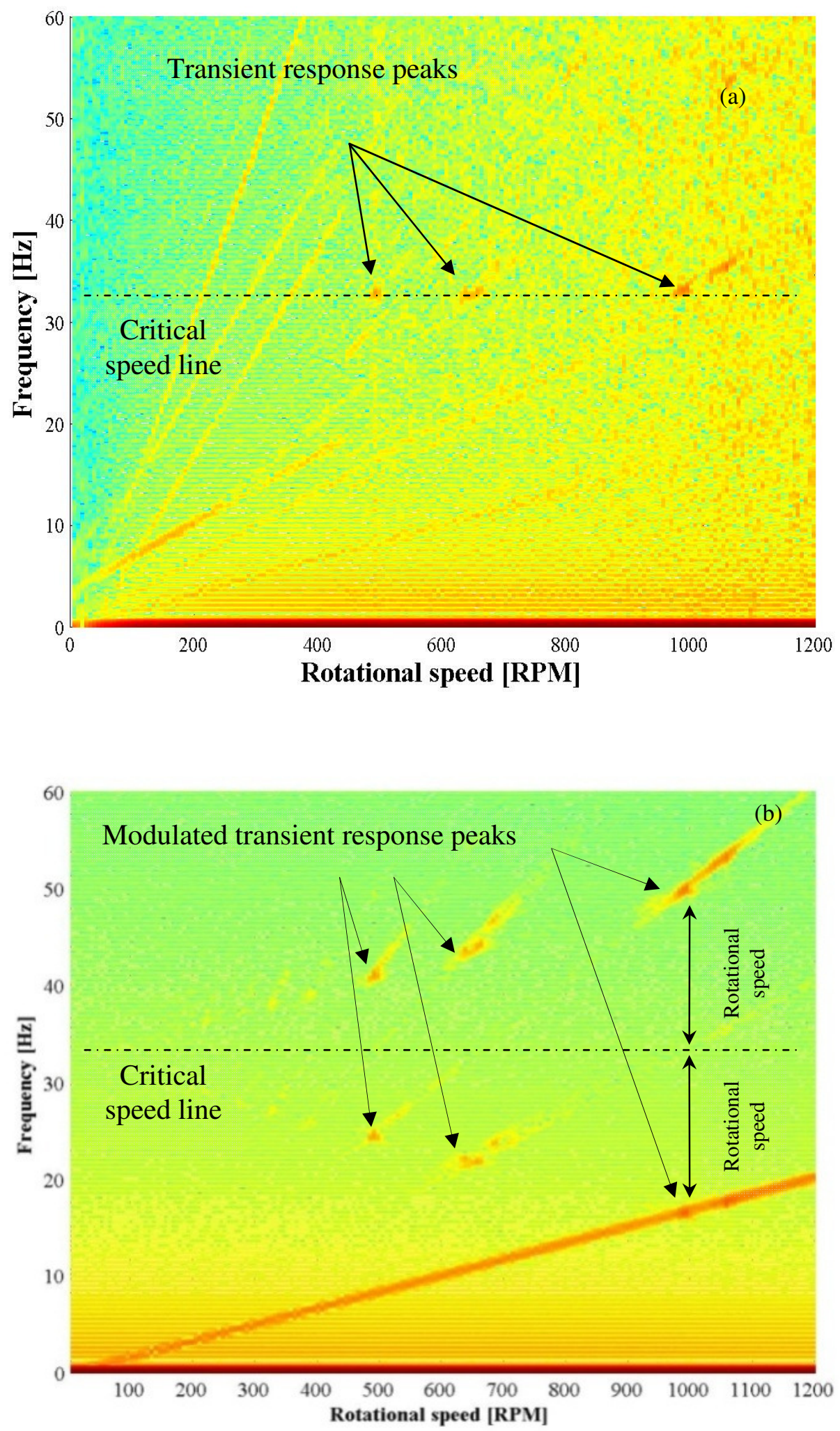

Figure 5: Spectrogram for the run up for (a) on-bearing and (b) on-shaft signals. 
(a)

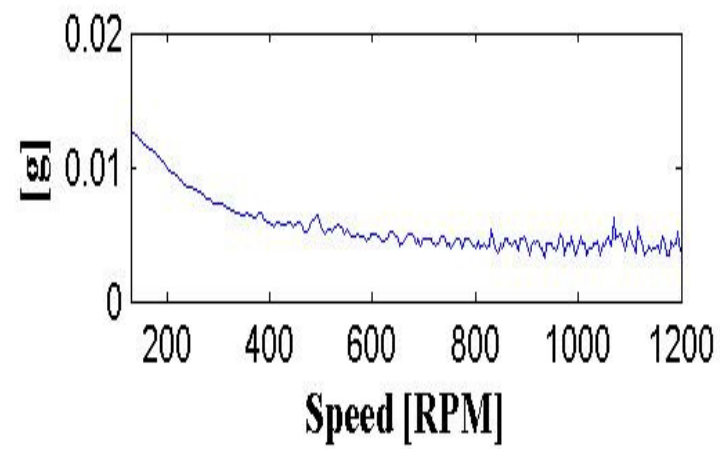

(b)

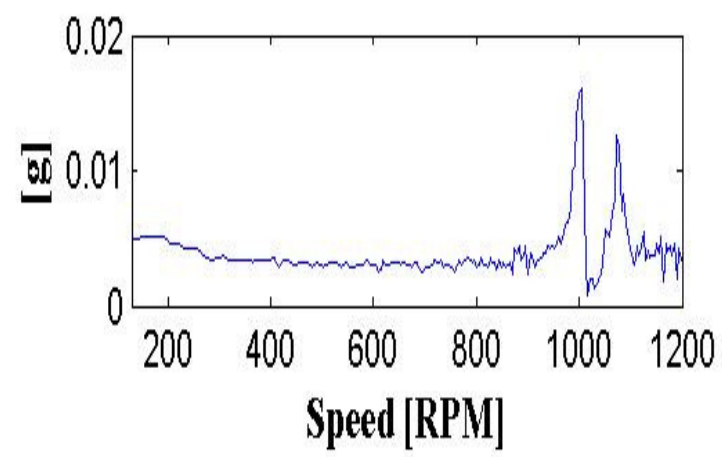

(c)

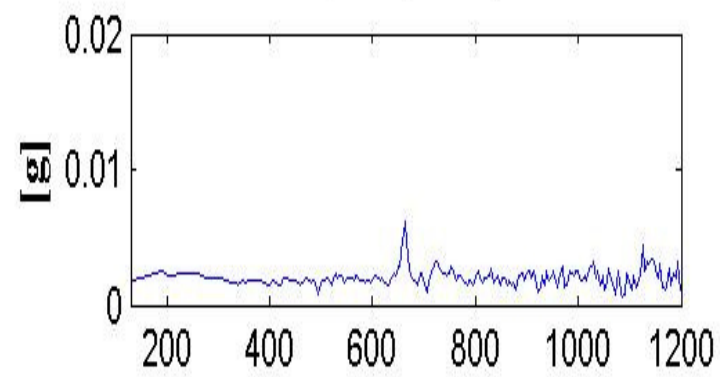

Speed [RPM]

(d)

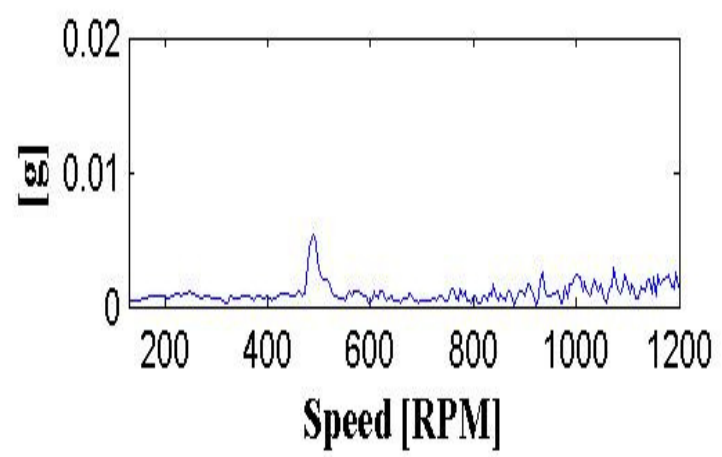

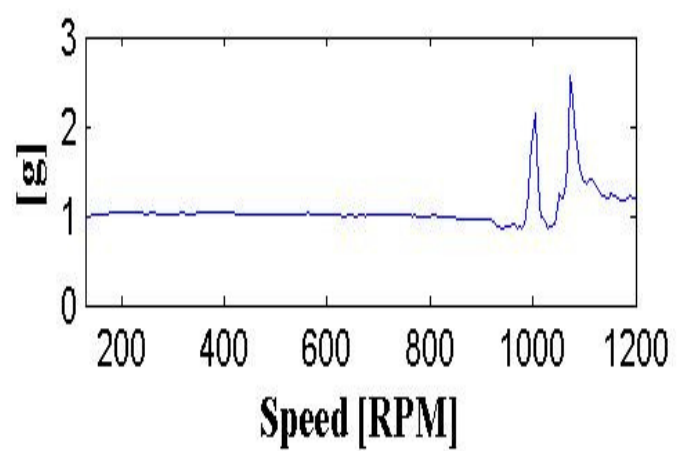
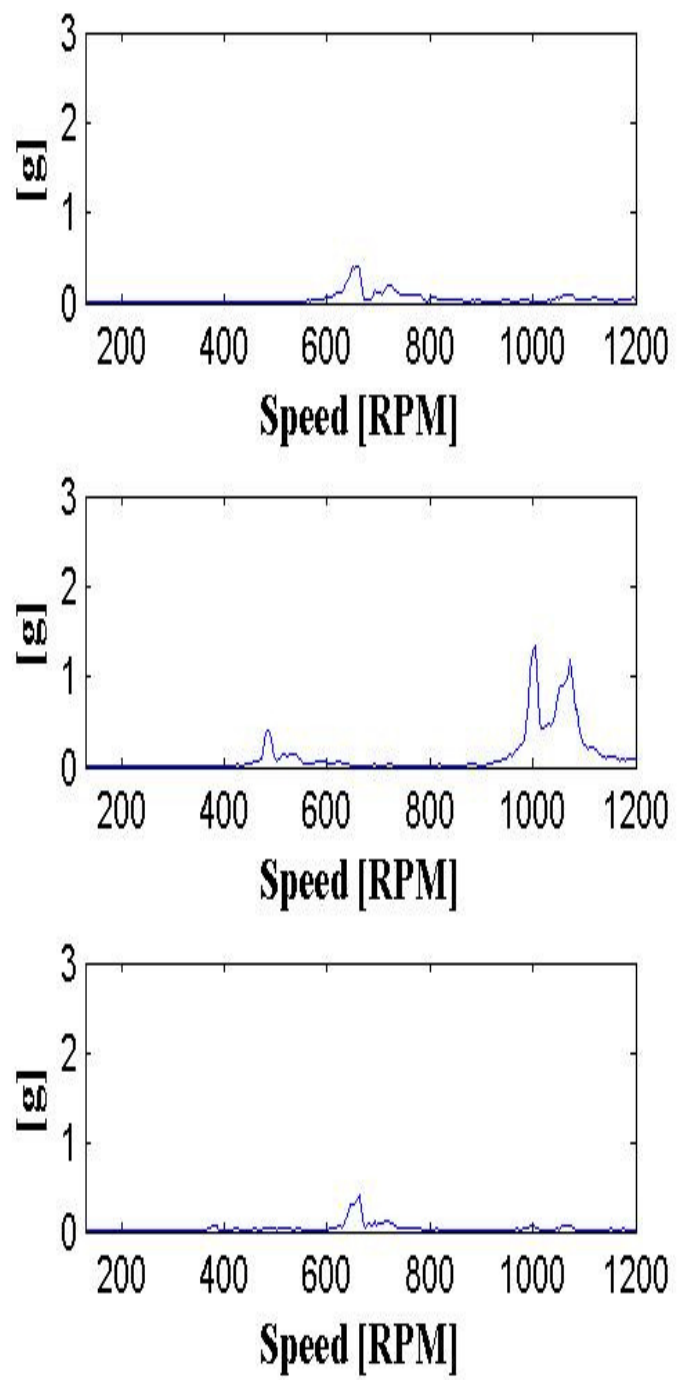

Figure 6: Effect of increasing speed on the on-bearing (left column) and on-shaft (right column) harmonics; (a) 1x, (b) 2x, (c) 3x, and (d) 4x. 


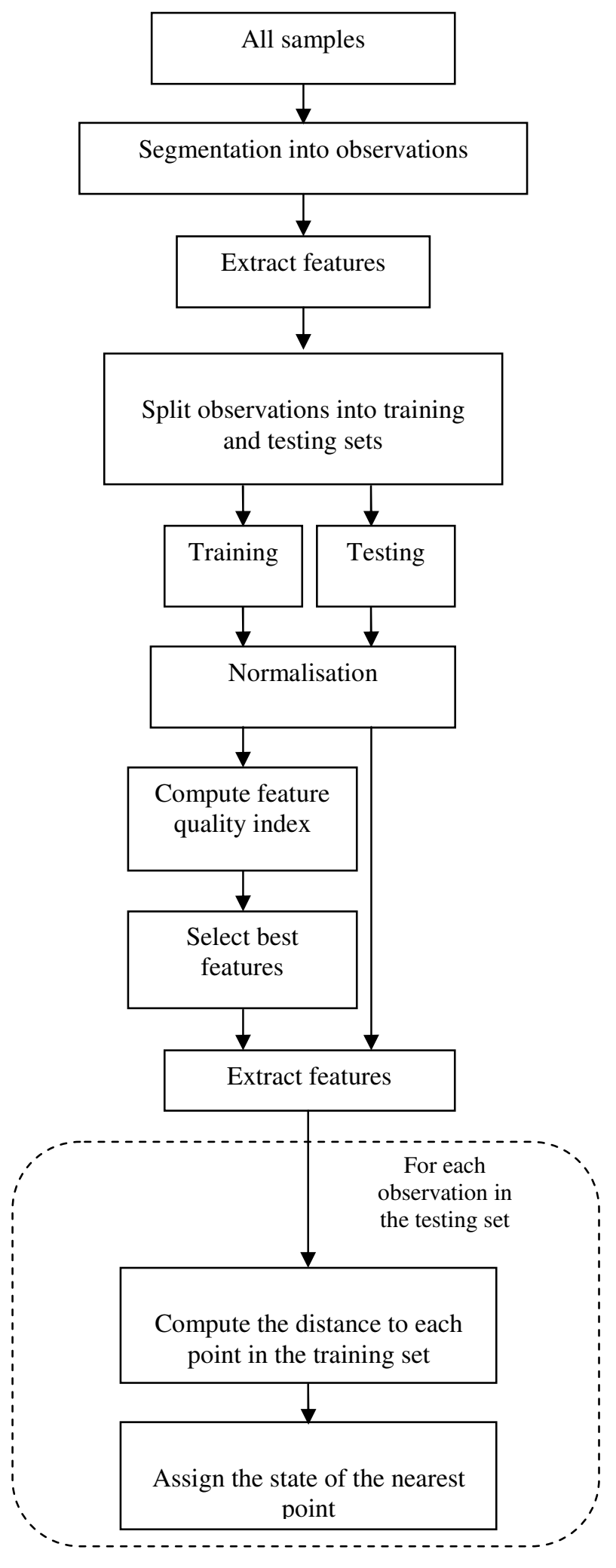

Figure 7: Classification algorithm flow chart. 

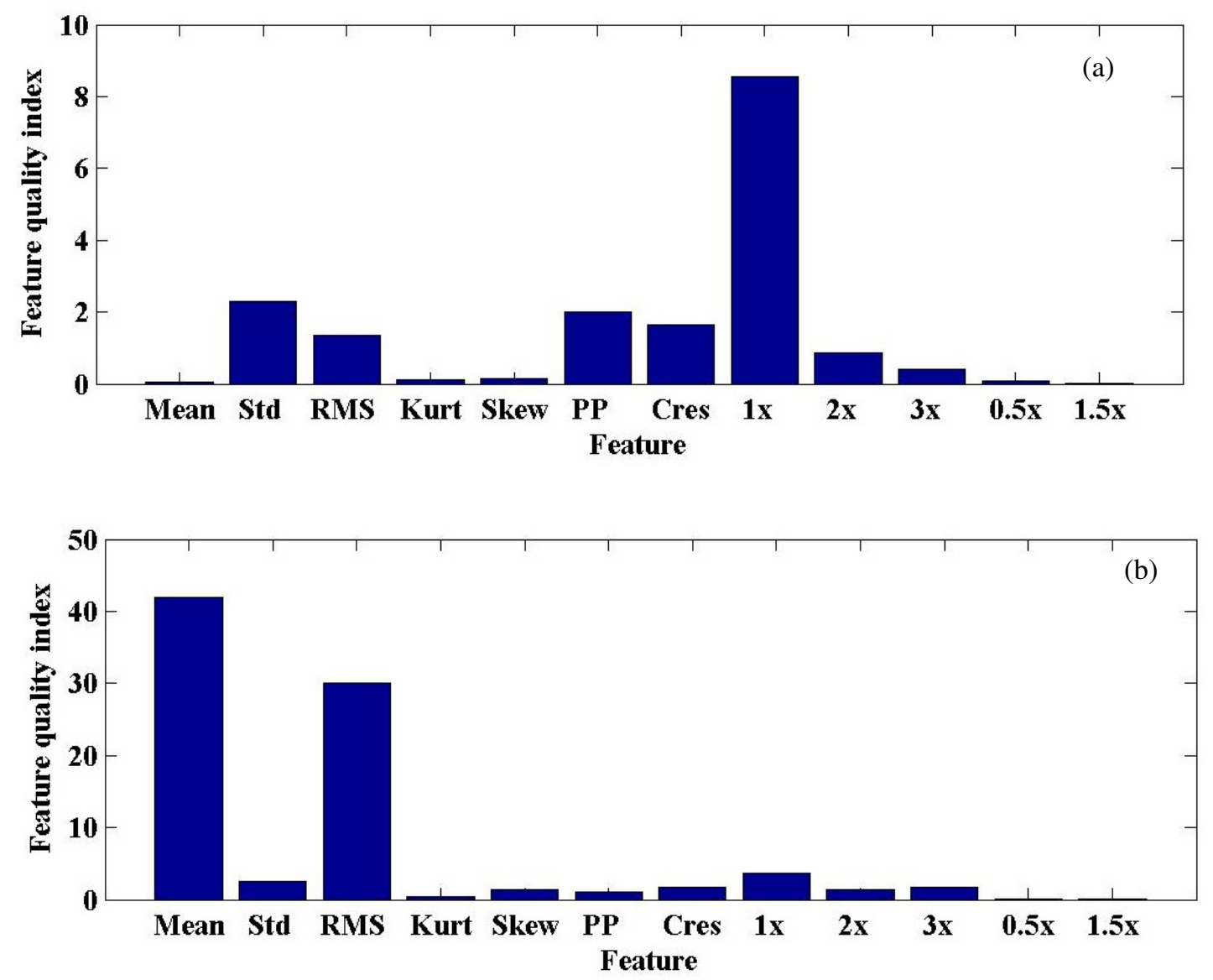

Figure 8: Feature Quality Index for (a) on-bearing and (b) on-shaft features.
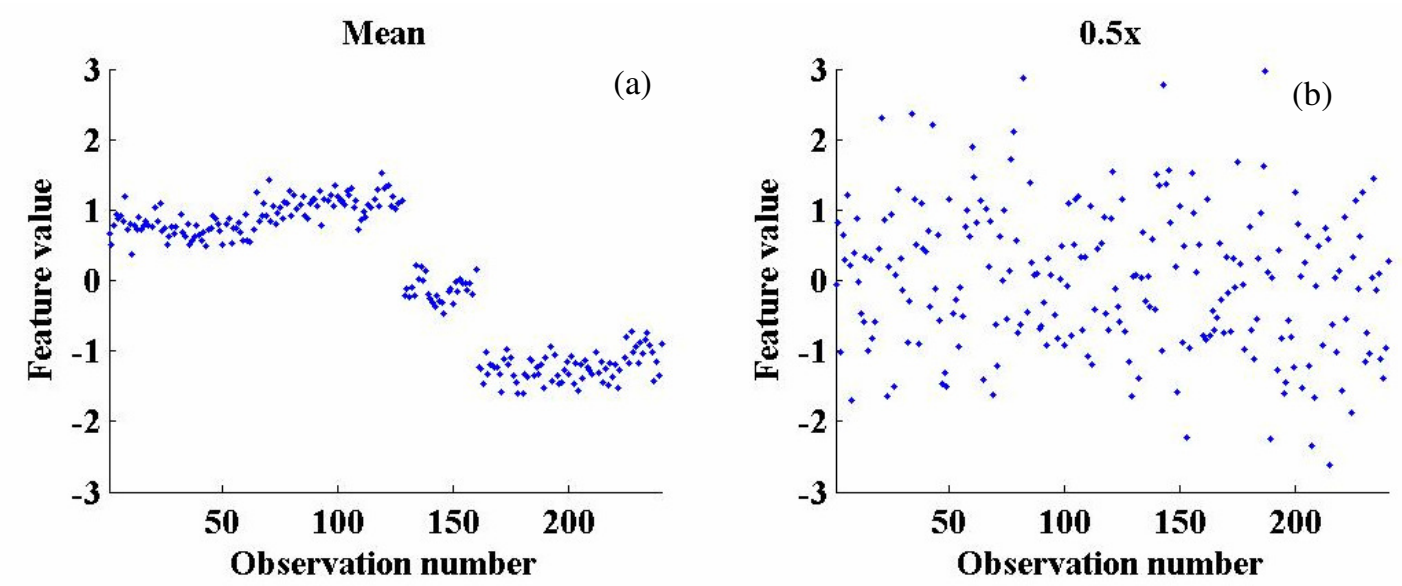

Figure 9: Features of (a) high and (b) low quality indices. 

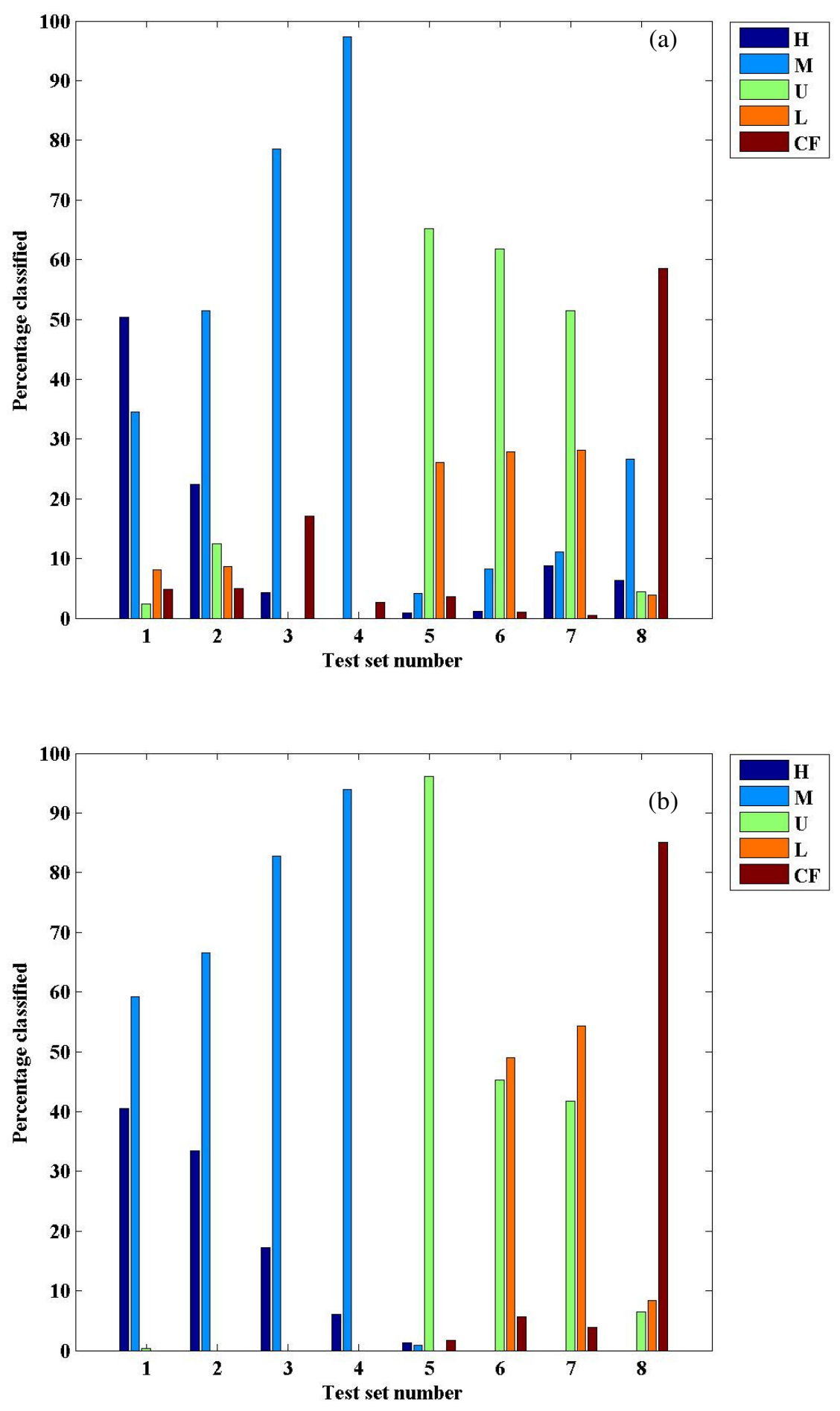

Figure 10: Classification results for (a) on-bearing and (b) on-shaft vibration measurement 

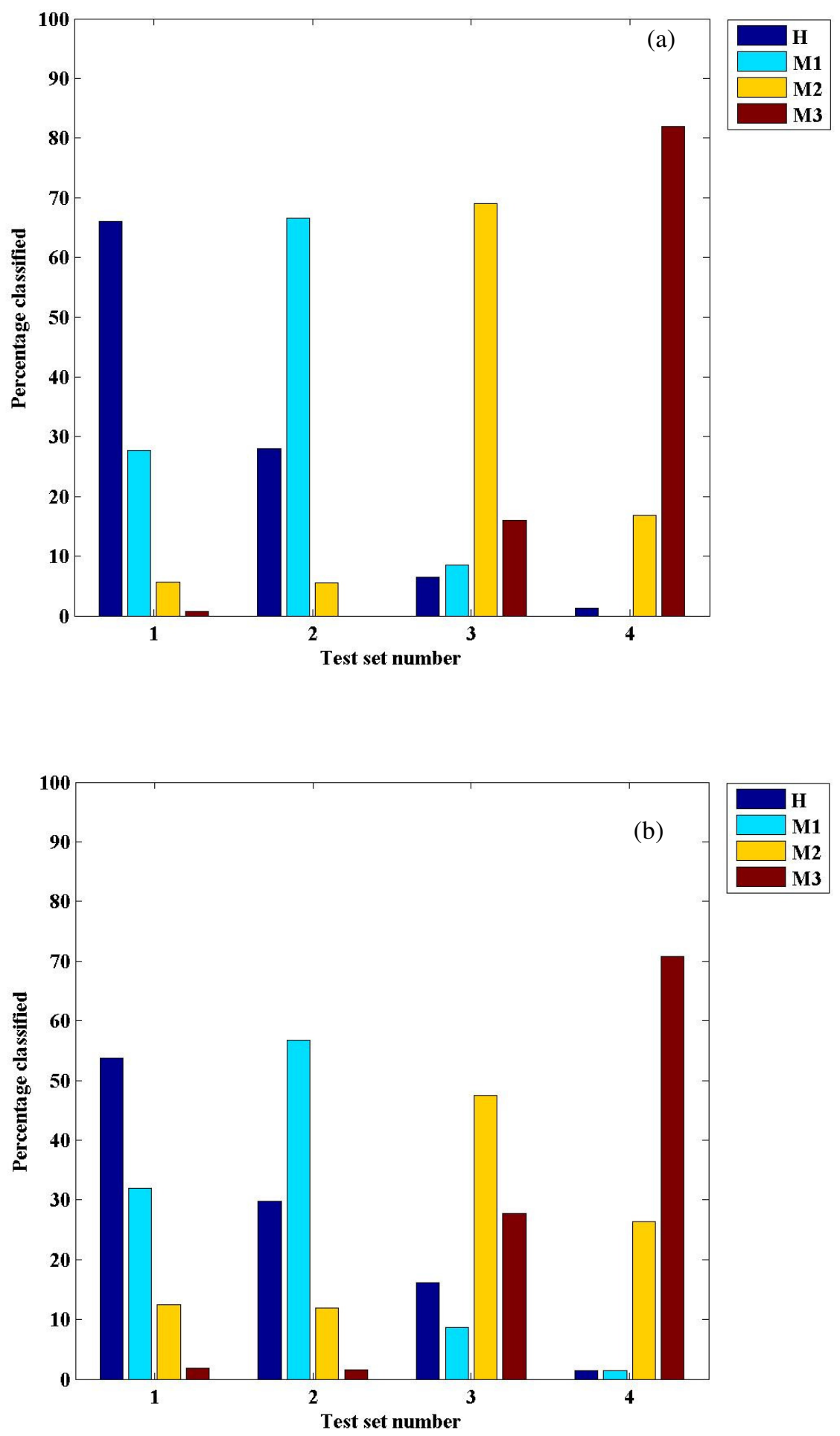

Figure 11: Classification results for different misalignment levels for (a) on-bearing and (b) on-shaft vibration measurement 

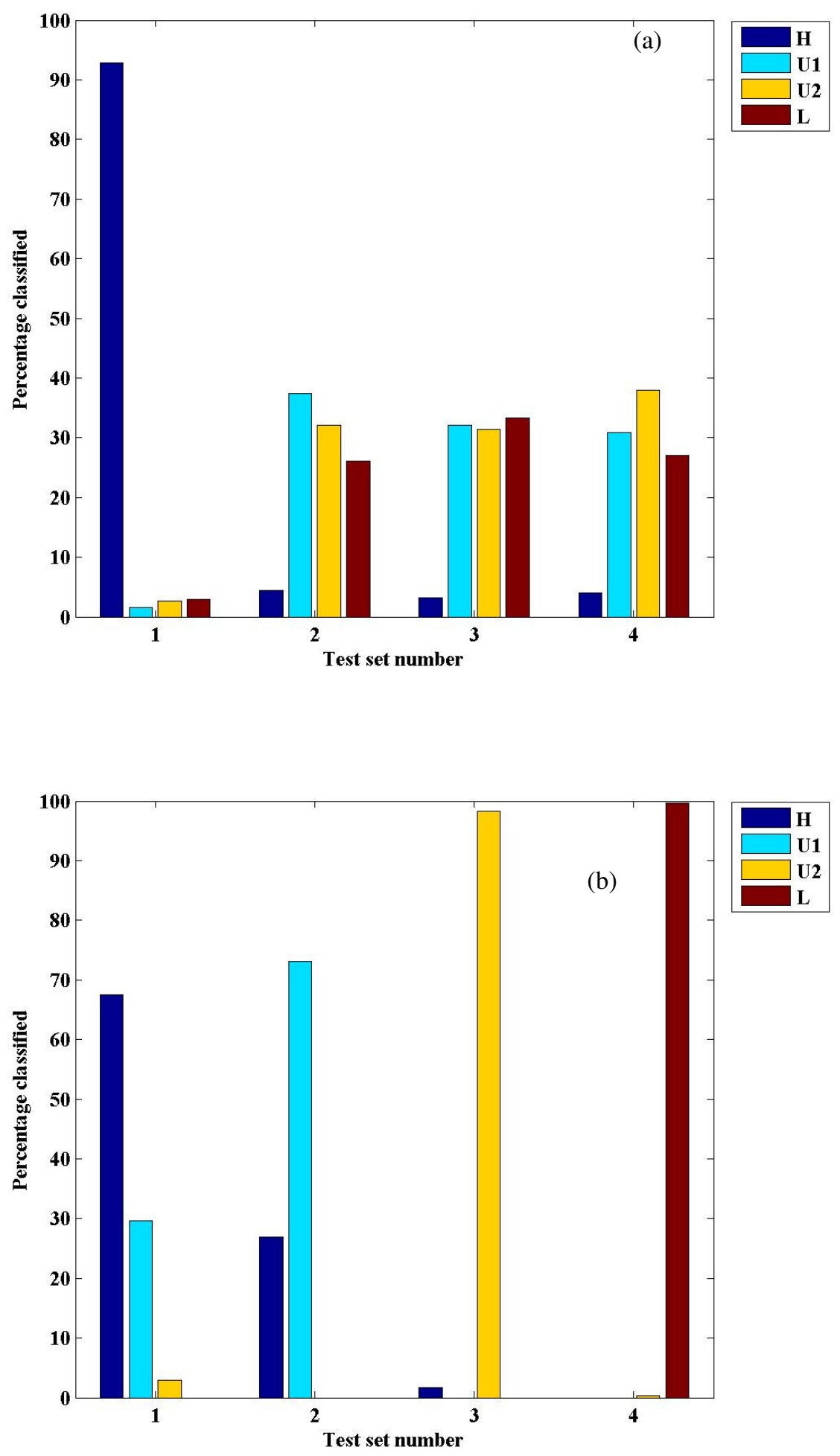

Figure 12: Classification results for different unbalance levels and looseness for (a) on-bearing and (b) on-shaft vibration measurement. 
Table 1. List of investigated machine states

\begin{tabular}{|c|c|c|c|c|}
\hline Set number & Machine state/fault & Fault level & Abbreviation & $\begin{array}{c}\text { Generalized } \\
\text { state abb. }\end{array}$ \\
\hline 1 & Reference & 1 & $\mathrm{R}$ & - \\
\hline 2 & \multirow{3}{*}{ Misalignment } & 1 & M1 & \multirow{3}{*}{$M$} \\
\hline 3 & & 2 & M2 & \\
\hline 4 & & 3 & M3 & \\
\hline 5 & \multirow{2}{*}{ Unbalance } & 1 & U1 & \multirow{2}{*}{$U$} \\
\hline 6 & & 2 & U2 & \\
\hline 7 & Looseness & 1 & L & - \\
\hline 8 & Combined fault & 1 & CF & - \\
\hline
\end{tabular}

Table 2. List of used time and frequency features

\begin{tabular}{|c|c|}
\hline Time domain features & Frequency domain features \\
\hline Mean & $1 \times$ component \\
\hline Standard deviation (Std) & $2 \times$ component \\
\hline Root mean square (RMS) & $3 \times$ component \\
\hline Kurtosis (Kurt) & $0.5 \times$ band \\
\hline Skewness (Skew) & $1.5 x$ band \\
\hline Peak to peak (PP) & \\
\hline Crest factor (Cres) & \\
\hline
\end{tabular}

